\section{Yalow, Rosalyn}

W. Hubl

Lebensdaten Amerikanische Physikerin, geboren am 19. Juli 1921 in New York City, gestorben am 30. Mai 2011 in New York City. Physikstudium in New York am Hunter College, Assistentenstelle für Physik an der Universität in Illinois. Im Jahr 1945 erwarb sie den Ph. D. auf dem Gebiet der Kernphysik. Von 1945-1946 arbeitete sie als Hilfsingenieurin vorübergehend im Forschungslabor der International Telephone and Telegraph Corporation. Am Bronx Veterans Administration Hospital begann hierauf die erfolgreiche 22-jährige Zusammenarbeit mit Solomon A. Berson (1918-1972) zunächst mit der Anwendung von Radioisotopen bei Patienten mit Schilddrüsenerkrankungen und danach mit der hormonellen Regulation des Magen-Darm-Trakts sowie der Differenzierung der Molekülarten des Gastrins ( $\triangleright$ Gastrin). Ab 1970 leitete sie die Abteilung für Nuklearmedizin am Bronx Hospital.

Ihr Mitstreiter Berson verstarb 1972, weshalb Rosalyn Yalow den Nobelpreis im Jahr 1977 ohne ihn entgegennahm. Sie stand von 1972-1992 dem Labor „Solomon A. Berson“ vor. Yalow übernahm von 1980-1985 den Vorsitz des Department of Clinical Science, Montefiore Hospital and Medical Center. Danach emeritierte sie als Emeritus Professor, Albert Einstein College of Medicine, Yeshiva University.

Verdienste Im Jahr 1975 wurden Yalow und Berson (posthum) mit dem American Medical Association (AMA) Scientific Achievement Award ausgezeichnet. Sie erhielt im Jahre 1976 als erste weibliche Wissenschaftlerin den Albert Lasker-Preis für Basic Medical Research. Im Jahr 1978 wurde sie zum Fellow of the American Academy of Arts and Sciences gewählt und erhielt 1988 die National Medal of Science.
1977 erhielt Yalow den Nobelpreis für Medizin gemeinsam mit Roger Guillemin (geb. 1924), USA, und Andrew Victor Schally (geb. 1926), USA, „Für die Entwicklung radioimmunologischer Methoden der Bestimmung von Peptidhormonen“ ( $\triangleright$ Peptidhormone), und die andere Hälfte „Für die Entdeckungen der Peptidhormone im Gehirn“ ging an Guillemin und Schally. Ihre größte Entdeckung war der $>$ Radioimmunoassay für $>$ Insulin, mit dem es möglich wurde, geringste Konzentrationen im Blut nachzuweisen. Diese $\mathrm{Pu}$ blikation aus dem Jahr 1959 sowie die Möglichkeit der universellen Anwendung dieses Verfahrens zur Bestimmung nahezu aller biologisch wirksamen Substanzen im Organismus in kleinsten Konzentrationen (Hormone, Peptide, Proteine, Enzyme, Vitamine, Medikamente etc.) erregte weltweites Aufsehen und leitete die Ära des Radioimmunoassays ein.

\section{Literatur}

Glick S (2011) Rosalyn Sussman Yalow (1921-2011). Nature 474:580 Herder WW (2014) Heroes in endocrinology: nobel prizes. Endocr Connect 3:R99

Yalow RS, Berson SA (1959) Assay of plasma insulin in human subjects by immunological methods. Nature 184:1648-1649

\section{Yersinia enterocolitica}

W. Stöcker

Englischer Begriff Yersinia enterocolitica

Beschreibung des Erregers Familie: Enterobacteriaceae; Gattung: Yersinia $(Y$.$) .$ 
Die zur Familie der Enterobacteriaceae gehörende Gattung Yersinia umfasst derzeit 14 Spezies. Obligat humanpathogen sind Y. pestis, Y. pseudotuberculosis und Y. enterocolitica. Die Spezies $Y$. enterocolitica bildet eine heterogene Gruppe von pathogenen und apathogenen Stämmen, die in über 60 verschiedene Serovare subdifferenziert werden können.

Mikroskopisch stellen sich die Bakterien als gramnegative, kokkoide bis pleomorphe, meist alkalistabile, psychrophile (kälteliebende) Kurzstäbchen mit mono- bis peritricher Begeißelung dar.

Erkrankungen $Y$. enterocolitica ist weltweit in den gemäBigten und subtropischen Klimazonen verbreitet. Das Bakterium kommt bei vielen warmblütigen Wild-, Heim-, und Nutztieren im Darm (selten Rachen), in ihren Ausscheidungen sowie in der Umwelt vor. Schweine stellen das wichtigste Reservoir für die menschliche Infektion dar. Die Infektion erfolgt über nicht ausreichend erhitzte tierische Produkte, hauptsächlich rohes Schweinefleisch und Milch. Auch kontaminierte Blutkonserven sowie der direkte Umgang mit Schweinen oder Haustieren stellen ein Infektionsrisiko dar.

Die durch pathogene $Y$.-enterocolitica-Stämme hervorgerufene enterale Yersiniose des Menschen stellt in Deutschland eine der häufigsten bakteriell bedingten Magen-DarmInfektionen dar (3364 Fälle im Jahr 2010). Sekundär können extraintestinale, immunologisch bedingte Reaktionen, wie Erythema nodosum, Uveitis, reaktive Arthritis (Morbus Reiter), Glomerulonephritis, Thyreoiditis oder Myokarditis auftreten.

Zur Prophylaxe einer Infektion sind in erster Linie die Einhaltung hygienischer Standards bei der Lebensmittelherstellung und -zubereitung zu nennen. Die Behandlung einer akuten Infektion beschränkt sich in der Regel auf symptomatische Maßnahmen wie den Ersatz der Flüssigkeits- und Salzverluste, die durch das Erbrechen und den Durchfall entstehen. Als unterstützende Maßnahmen können Medikamente eingesetzt werden, die das Erbrechen hemmen oder die Darmtätigkeit beeinflussen. In schweren Fällen kann bei positivem Erregernachweis eine Antibiotikatherapie erfolgen (Breitspektrum-Cephalosporin plus Aminoglykosid).

Analytik Der Erregernachweis erfolgt durch die Anzucht aus Stuhl und nicht fäkalen Proben wie Blut oder Biopsien auf selektiven Nährmedien. Bei einer zu geringen Anzahl an Bakterien in der Probe wird zunächst eine Kälteanreicherung $\left(4^{\circ} \mathrm{C}, 1-3\right.$ Wochen) durchgeführt. Aufgrund der Heterogenität innerhalb der Spezies sind die biochemische Bestätigung und Biotypisierung sowie die serologische Pathogenitätsbestimmung der Isolate zur Identifikation virulenter Stämme wichtig. Der direkte Erregernachweis mit molekularbiologischen Methoden ( $\triangleright$ PolymeraseKettenreaktion) gewinnt zunehmend an Bedeutung, insbesondere weil die Pathogenitätsgene auf diesem Wege mit identifiziert werden können. Allerdings ist auch hier wegen der Begleitflora zunächst eine selektive Anzucht von Vorteil.

Eine Yersinien-Infektion induziert die Bildung spezifischer Serumantikörper der Immunglobulinklassen IgA, IgG und IgM (s. > Immunglobuline), für deren Nachweis ein auf Virulenzfaktoren (Yop D, E, H, M) basierender \ Immunblot, Immunfluoreszenztest ( $\triangleright$ Immunfluoreszenz, indirekte) und $\checkmark$ Enzymimmunoassay eingesetzt werden. Weitere Nachweisverfahren sind Komplementbindungsreaktion und Widal-Reaktion. Yersiniosen sind nach $\S 7$ Abs. 1 Infektionsschutzgesetz meldepflichtig.

\section{Untersuchungsmaterial - Probenstabilität Kultur und} PCR: Stuhl, Blut, Biopsie, Lymphknotenabstrich.

Serologie: Serum oder Plasma für den Nachweis der Antikörper sind bei $+4{ }^{\circ} \mathrm{C}$ bis zu 2 Wochen beständig, bei $-20^{\circ} \mathrm{C}$ über Monate und Jahre hinweg. Zur Tiefkühlkonservierung des IgM kann man den Proben 80 \% gepuffertes Glyzerin beifügen.

Diagnostische Wertigkeit Für die Diagnose einer akuten Infektion mit Yersinia enterocolitica ist der direkte Erregernachweis im Stuhl die Methode der Wahl. Der Antikörpernachweis wird hauptsächlich zur Abklärung von Yersinien-assoziierten Folgeerkrankungen, vor allem der reaktiven Arthritis, eingesetzt.

\section{Literatur}

Robert-Koch-Institut Berlin, Epidemiologisches Bulletin, 13. Februar 2012 / Nr. 6. Aktuelle Daten und Informationen zu Infektionskrankheiten und Public Health. Yersiniose-Risikofaktoren in Deutschland Tschäpe H, Reissbrodt R, Prager R (2009) Yersinia ssp. In: Neumeister B, Geiss HK, Braun RW, Kimmig P (Hrsg) Mikrobiologische Diagnostik, 2. Aufl. Thieme, Stuttgart/New York, S 454-457

\section{Yk $k^{a}$-Antigen}

- York-Antigen

YKL-40

A. M. Gressner und O. A. Gressner

Synonym(e) Chondrex

Englischer Begriff human cartilage glycoprotein-39 (HC gp39); 38 kDa heparin-binding-glycoprotein

Definition YKL-40 ist ein in zahlreichen Geweben und Organen exprimiertes Glykoprotein, das unter entzündlichen 
und malignen Bedingungen überexprimiert und in erhöhten Konzentrationen im Serum gemessen wird.

Beschreibung Humanes YKL-40 ist ein lineares Glykoprotein von $40 \mathrm{kDa}$ mit bekannter Sequenz der 383 Aminosäuren. Die Bezeichnung leitet sich von seinen 3 N-terminalen Aminosäuresequenzen $\mathrm{ab}$ : Tyrosin $=\mathrm{Y}$, Lysin $=\mathrm{K}$, Leucin $=\mathrm{L}$. Es gehört zur Familie der Glykosylhydrolasen 18, die signifikante Sequenzhomologien zu bakteriellen Chitinasen aufweisen. $\mathrm{Zu}$ den Komponenten der extrazellulären Matrix $($ ECM) $>$ Fibronectin, Kollagenen der Typen I, II, III und IV ( $>$ Kollagene) sowie $>$ Hyaluronan bestehen enge Bindungen und Wechselwirkungen. Seine Funktion ist noch weitgehend unbekannt. YKL-40 zeigt eine ausgeprägte Verteilung in Zellen und Geweben: Osteoblasten, Chondrozyten, Synovialzellen ( $>$ Synovia-Analyse), vaskuläre glatte Muskelzellen, aktivierten $>$ Makrophagen, neutrophile Granulozyten, neurale Retinazellen, hepatische Stellat-(Stern-)Zellen (Ito-Zellen, $>$ Fibrosekenngrößen), aber nicht in Hepatozyten. Dieser breiten zellulären Verteilung entspricht die Überexpression von YKL-40 unter vielen pathologischen Bedingungen wie chronische Entzündungen und fibrotische Organveränderungen: Leberfibrose, -zirrhose, rheumatoide Arthritis, Osteoarthritis, ulzerative Kolitis, Morbus Crohn, chronisch obstruktive pulmonale Erkrankung (COPD). In zahlreichen Karzinomen ist eine verstärkte Expression von YKL-40 immunhistologisch und durch In-situ-Hybridisierung nachweisbar. YKL-40 fördert die Metastasierung, Invasivität und Migration einiger Tumorarten und greift in die epitheliale mesenchymale Transition (EMT) ein. Proinflammatorische $>$ Zytokine wie $>$ Interleukin-1 (IL-1), IL-6 ( Interleukin-6), IL-13, TNF-alpha ( $\triangleright$ Tumornekrosefaktor- $\alpha$ ) und Hormone (z. B. Vasopressin) stimulieren die YKL-Synthese.

YKL-40 ist in Körperflüssigkeiten (Serum, Plasma, Synovialflüssigkeit, > Liquor cerebrospinalis, zellkonditionierten Medien) immunologisch (ELISA) quantifizierbar. Unter den beschriebenen Bedingungen wie Tumoren, Entzündungen und Malignomen sind signifikant erhöhte Konzentrationen im Serum messbar.

\section{Literatur}

Jefri M, Huang YN, Huang WC, Tai CS, Chen WL (2015) YKL-40 regulated epithelial-mesenchymal transition and migration/invasion enhancement in non-small cell lung cancer. BMC Cancer 15:590-612

Johansen JS (2006) Studies on serum YKL-40 as a biomarker in diseases with inflammation, tissue remodeling, fibroses and cancer. Dan Med Bull 53:172-208

\section{Yo-Antikörper}

- Autoantikörper gegen Yo

\section{York-Antigen}

K. Kleesiek, C. Götting, J. Diekmann, J. Dreier und M. Schmidt

Synonym(e) COST3; ISBT 022.005; KN5; Yk ${ }^{\mathrm{a}}$-Antigen

Englischer Begriff York antigen

Definition Das $\mathrm{Yk}^{\mathrm{a}}$-Antigen gehört in das $>$ Knops-Blutgruppensystem und wird auf $>$ Erythrozyten exprimiert (Antigenfrequenz: $92 \%$ Kaukasier, $98 \%$ Menschen mit dunkler Hautfarbe). Antikörper der Spezifität Anti-Yk ${ }^{\mathrm{a}}$ gehören zu den HTLA-Antikörpern (s. $>$ HTLA-Antikörper).

\section{Youden-Diagramm}

\section{R.-D. Hilgers, N. Heussen und S. Stanzel}

\section{Englischer Begriff Youden plot}

Definition Graphische Zusammenfassung der Ergebnisse vom einem $>$ Ringversuch.

Schematische Darstellung eines Youden-Diagramms:

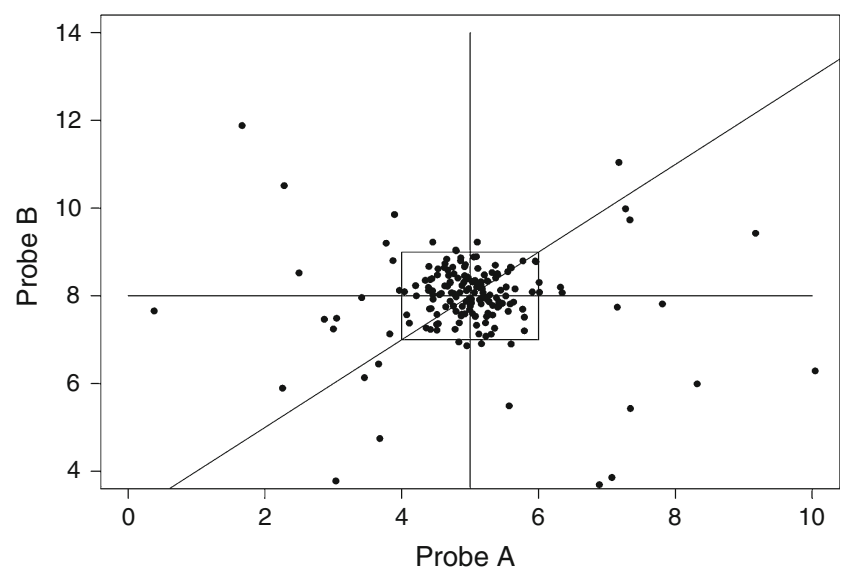

Beschreibung Die Messergebnisse (s. Messergebnis) der Probe A werden auf der Abszisse (x-Achse), die Ergebnisse der Probe B auf der Ordinate (y-Achse) abgetragen. Jedes Laboratorium liefert ein Wertepaar (ein Wert für Probe A, ein Wert für Probe B; jedem Wertepaar entspricht ein Punkt im Koordinatensystem). Das Youden-Diagramm ermöglicht jedem teilnehmenden klinisch-chemischen Labor einen Vergleich seiner Messergebnisse mit denen der anderen teilnehmenden Laboratorien. Darüber hinaus liefert das Youden-Diagramm Hinweise 
Yt-Blutgruppensystem, Tab. 1 Eigenschaften der Hauptantigene des Yt-Blutgruppensystems

\begin{tabular}{|c|c|c|c|c|c|c|c|}
\hline \multirow[b]{2}{*}{ Yt-Antigen } & \multirow{2}{*}{$\begin{array}{l}\text { ISBT- } \\
\text { Symbol } \\
\text { (Zahl) }\end{array}$} & \multirow[b]{2}{*}{$\begin{array}{l}\text { Antigenfrequenz } \\
(\%)^{*}\end{array}$} & \multicolumn{3}{|c|}{ In-vitro-Charakteristika von Alloantikörpern } & \multicolumn{2}{|c|}{$\begin{array}{l}\text { Klinische Signifikanz von } \\
\text { Alloantikörpern }\end{array}$} \\
\hline & & & Immunglobulinklasse & $\begin{array}{l}\text { Optimale } \\
\text { Nachweistechnik }\end{array}$ & Komplementbindung & Transfusionsreaktion & MHN \\
\hline $\begin{array}{l}\mathrm{Yt}^{\mathrm{a}} \text {, } \\
\text { Cartwright }\end{array}$ & $\begin{array}{l}\text { YT1 } \\
(011.001)\end{array}$ & 99,8 & IgG & IAT & Nein & $\begin{array}{l}\text { Keine bis moderate/ } \\
\text { verzögert }\end{array}$ & Keine \\
\hline $\mathrm{Yt}^{\mathrm{b}}$ & $\begin{array}{l}\text { YT2 } \\
(011.002)\end{array}$ & 8 & IgG & IAT & Nein & Nicht bekannt & Keine \\
\hline
\end{tabular}

*In allen Populationen; IAT, indirekter Agglutinationstest; ISBT, International Society of Blood Transfusion; MHN, Morbus haemolyticus neonatorum

auf systematische und zufällige Fehler ( $\triangleright$ Messabweichung, systematische; $>$ Fehler, zufälliger). Das umrandete Feld im Inneren des Diagramms repräsentiert den Bereich 1 Standardabweichung in $\mathrm{x}$ - und $\mathrm{y}$-Richtung.

\section{Literatur}

Youden WJ (1959) Graphical diagnosis of interlaboratory test results Ind Qual Control 15:24-28

\section{Yq11}

Azoospermiefaktor-Mutation

\section{Yt-Blutgruppensystem}

K. Kleesiek, C. Götting, J. Diekmann, J. Dreier und M. Schmidt

Synonym(e) ACHE; Cartwright

Englischer Begriff Yt blood group system
Definition Das Enzym Acetylcholinesterase (ACHE), das Produkt des ACHE-Gens (YT), trägt die Antigene des Yt-Blutgruppensystems.

Beschreibung ACHE wird als Vorläufer aus 585 Aminosäuren synthetisiert. Mit der Erythrozytenmembran ist dieses Glykoprotein über einen GPI-Anker (,glycosyl phosphadityl inositol") verbunden. Das N- und O-glykosylierte Protein kommt in der Erythrozytenmembran wahrscheinlich als Dimer vor ( $\triangleright$ Blutgruppensysteme). Das ACHE-Gen wird in neuralen und erythroiden Geweben exprimiert. Es kommt in Erythrozyten, Granulozyten und in Hirn- und Muskelgewebe vor. Die Funktion der Acetylcholinesterase in Erythrozyten ist nicht bekannt. Dieses Enzym katalysiert die hydrolytische Spaltung des Neurotransmitters Acetylcholin (ACh) in Acetat und Cholin. Patienten mit paroxysmaler nächtlicher Hämoglobinurie ( $\mathrm{PNH}$ ) haben eine Population von Komplement-sensitiven Erythrozyten (PNHIII), denen alle GPI-Anker-Glykoproteine, inklusiv der Acetylcholinesterase fehlen. Die Yt-Antigene (Tab. 1) können zur Bildung von erythrozytären Antikörpern führen, die zur Gruppe der > HTLA-Antikörper zählen.

\section{Literatur}

Blood Group Antigen Gene Mutation Database, NCBI National Center of Biotechnology Information, Bethesda, Maryland

Reid ME, Lomas-Francis C (2004) The blood group antigen facts book, 2. Aufl. Elsevier, New York 